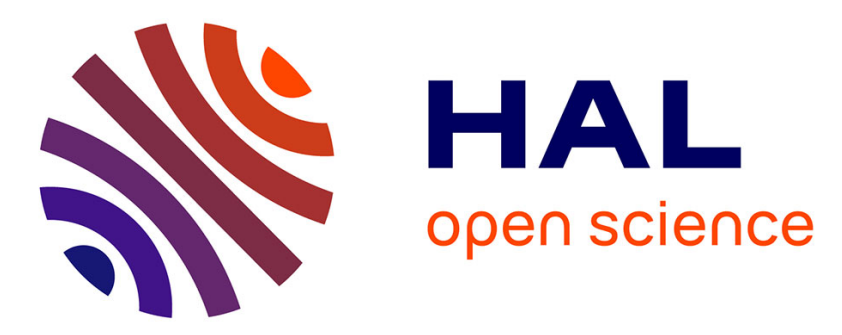

\title{
Handling Continuous Functions in Hybrid Systems Reconfigurations: A Formal Event-B Development
}

Guillaume Babin, Yamine Aït-Ameur, Neeraj Kumar Singh, Marc Pantel

\section{To cite this version:}

Guillaume Babin, Yamine Aït-Ameur, Neeraj Kumar Singh, Marc Pantel. Handling Continuous Functions in Hybrid Systems Reconfigurations: A Formal Event-B Development. ABZ 2016 - 5th International Conference Abstract State Machines, Alloy, B, TLA, VDM, and Z, May 2016, Linz, Austria. pp.290-296, 10.1007/978-3-319-33600-8_23 . hal-03155064

\section{HAL Id: hal-03155064 https://hal.science/hal-03155064}

Submitted on 2 Mar 2021

HAL is a multi-disciplinary open access archive for the deposit and dissemination of scientific research documents, whether they are published or not. The documents may come from teaching and research institutions in France or abroad, or from public or private research centers.
L'archive ouverte pluridisciplinaire HAL, est destinée au dépôt et à la diffusion de documents scientifiques de niveau recherche, publiés ou non, émanant des établissements d'enseignement et de recherche français ou étrangers, des laboratoires publics ou privés. 


\title{
Handling Continuous Functions in Hybrid Systems Reconfigurations: A Formal Event-B Development
}

\author{
Guillaume Babin $^{(凶)}$, Yamine Aït-Ameur, Neeraj Kumar Singh, \\ and Marc Pantel \\ IRIT / INPT-ENSEEIHT, Université de Toulouse, \\ 2 rue Charles Camichel, Toulouse, France \\ guillaume.babin@irit.fr, \{yamine,nsingh,marc.pantel\}@enseeiht.fr
}

\begin{abstract}
This paper presents a substitution mechanism for systems having a continuous behavior. It shall preserve the safety property stating that the output of both systems remain in a safety envelope. The whole approach is formalized using Event-B, and relies on the Rodin tools and a theory of Reals provided by the Rodin Theory Plug-in to check the internal consistency with respect to safety properties, invariants and events.
\end{abstract}

\section{Introduction}

This paper relies on stepwise refinement in Event-B [2] to contribute to the formalization and verification of controllers in Cyber Physical Systems, relying on a generic substitution mechanism. In this work, we show how to apply the defined substitution as a reconfiguration mechanism to handle hybrid systems characterized by continuous functions which can be solutions of differential equations In this case as we model elements from the physical world, system substitutions are not instantaneous. We extensively use our previous work [4] on discrete controllers synthesis from continuous behavior descriptions. The primary use of the models is to assist in the construction, clarification, and validation of the continuous behavior controller requirements to build a digital controller in the presence of system reconfiguration or system substitution. In this development, we use the Rodin Platform [3,9] to manage model development, refinement, proofs checking, verification and validation.

\section{The Event-B Method}

An Event-B model [2] is defined by contexts and machines. It encodes a statetransitions system which consists of: variables to represent the state; and events to represent the transitions (defined by before-after predicates). A model also contains invariants to represent its relevant properties. A variant may ensure convergence properties when needed. An Event-B machine can see contexts that 
contain the relevant sets, constants and axioms. Refinement allows to add more behavior properties and system requirements by refining the abstract model to a more concrete one. New variables and new events may be introduced at the refinement level. Once a machine is defined, generated proof obligations need to be proven in order to demonstrate the preservation of the invariants.

Use of Reals in Event-B. In our work, we are interested in formalizing and analyzing system specifications while using reals. Therefore, we rely on the theory for reals, written by Abrial and Butler ${ }^{1}$. It provides a dense mathematical $R E A L$ datatype with arithmetic operators, axioms and proof rules.

\section{Our Previous Work}

This section recalls the seminal results we obtained for both system substitution and discretization of continuous functions. The work presented in this section is detailed in [4] and all the Event-B models related to the discretization of continuous functions are available in [1].

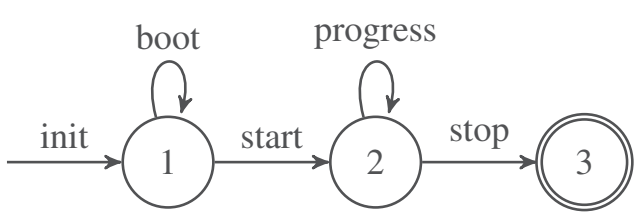

Fig. 1. Behavior of studied systems

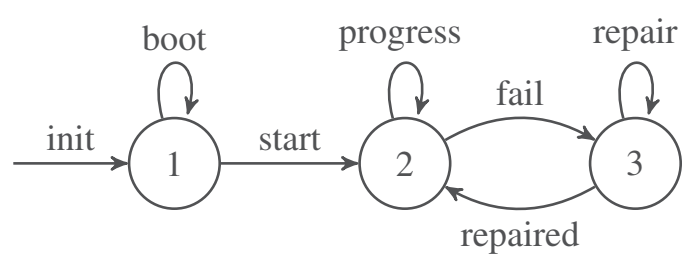

Fig. 2. System substitution

Studied Systems. They are formalized as state-transition systems. The behavior of such systems can be characterized by two states: the initial one (usually called boot) and the nominal one (progress). According to Fig. 1, after initialization, a system enters into the booting state (state 1). Then, after a start transition, the system progresses (state 2, known as running state). If the system stops, it switches to state 3 .

System Substitution [5,6]. One of the key properties studied in system engineering is the capability of a system to react to changes. It may occur in different situations (e.g. failures, change of quality of service, context evolution, maintenance, etc.). System substitution can be defined as replacing a system by another one while preserving the required behavior.

In $[5,6]$, we have developed a formal model (pattern) for system substitution described in the Event-B modeling language. This pattern is depicted in Fig. 2. When a failure occurs, the running system is halted (fail transition), it is repaired in state 3 where the state of the substitute system is restored from the one of the

\footnotetext{
${ }^{1}$ http://wiki.event-b.org/index.php/Theory_Plug-in\#Standard_Library.
} 
halted system. Finally, the control is restored to the substitute system (transition repaired). The correctness of this substitution has been studied in different cases (equivalent, degraded or upgraded ones). The defined substitution mechanism (Fig. 2) preserves the behavior of the original specification and restores correctly the state of the halted system.

Discretization of Continuous Functions [4]. To control a system, in particular for system reconfiguration, it is required to observe (monitor the feedback behavior of the function) and to control (maintain or change system mode) the system. Such observation and control is performed by a software requiring the discretization of continuous functions. When using computers to implement such controllers, time is observed according to specific clocks and frequencies. Therefore, it is mandatory to define a correct discretization of time that preserves the observed continuous behavior introduced previously. This preservation entails the introduction of other requirements on the defined continuous function. Note that, in practice, these requirements are usually satisfied by the physical plant.

In [4], we reported the stepwise formal development in Event-B of a correct discretization of a Lipschitz continuous function $f$ characterizing a hybrid system $S_{y} s_{f}$. The development consists in the following steps.

- Specification: the mode controller. It models systems whose behaviors are described in the state-transitions system of Fig. 1. The safety requirement ensures that the observed values remain in the safety envelope defined by the interval $[m, M]$.

- Introduction of continuous behaviors. This refinement consists in introducing a continuous function $f$ defined on the domain of positive real numbers (for dense time). The observed values of $f$ will belong to the defined safety envelope $[m, M]$.

- The second refinement: correct discretization of continuous behaviors. Discretization requires the introduction of a margin allowing the controller to anticipate (predictive control) the next observable behavior before incorrect behavior occurs. Let $z>0$ be this margin. We use the Lipschitz continuous function property of $f$ to define the amount of time between two consecutive states observed by the discrete controller. We introduced macro time steps of duration $\delta t$ (discrete control sampling time interval). As a consequence, we define $z$ such that $z \geq \max _{t \in \mathbb{R}^{+}}|f(t)-f(t+\delta t)|$. In order to make it well-defined, $\delta t$ must be small enough so that the property $m+z<M-z$ holds. As a consequence, the set $\mathbb{D}$ of observation instants can be defined as, $\mathbb{D}=\left\{t_{i} \mid t_{i} \in \mathbb{R} \wedge i \in \mathbb{N} \wedge t_{0}=0 \wedge t_{i+1}=t_{i}+\delta t\right\}$

It can be rewritten as $\mathbb{D}=\left\{t_{i} \mid t_{i} \in \mathbb{R} \wedge i \in \mathbb{N} \wedge t_{0}=0 \wedge t_{i}=i \times \delta t\right\}$. As a result of the definitions of $z$ and $\delta t$, if $f(n \times \delta t)$ is in $[m+z, M-z]$ then we can safely predict using the Lipschitz condition that the next value of $f$ observed by the controller, $f((n+1) \times \delta t)$, is in $[m, M]$. 


\section{Hybrid System Substitution in the Presence of Continuous Behaviors}

We consider two continuous functions $f$ and $g$ characterizing the behavior of two hybrid systems $S y s_{f}$ and $S y s_{g}$. We also assume that these two systems maintain their feedback information value in the safety envelope $[m, M]$. As a consequence, these two systems can substitute each other since they fulfill the same safety requirement. The studied scenario consists in substituting $S y s_{f}$ after a failure by $\mathrm{Sys}_{g}$ (see requirements in Table1).

Figure $3 \mathrm{a}$ and $\mathrm{b}$ show the substitution scenario in both continuous and discrete cases. The $\mathrm{X}$ axis describes time passing and the vertical dashed lines model state transitions according to the behavior depicted in Fig. 2. Observe that during repair (state 3 of Fig. 2), the function $f$ (associated with $S y s_{f}$ ) decreases while the function $g$ (associated with $S y s_{g}$ ) is booting. The invariant states that the function $f+g$ belongs to the safety envelope $[m, M]$ during the repair. Finally, the system returns to progress (state 2) using $S y s_{g}$ as the running system.

Table 1. Requirements in the abstract specification.

\begin{tabular}{l|l}
\hline At any time, the feedback information value of the controlled & Req.1 \\
system shall be less or equal to $M$ in any mode. & \\
\hline $\begin{array}{l}\text { At any time, the feedback information value of the controlled } \\
\text { system shall belong to an interval }[m, M] \text { in progress mode. }\end{array}$ & Req.2 \\
\hline $\begin{array}{l}\text { The system feedback information value can be produced } \\
\text { either by } f, g \text { or } f+g \text { ( } f \text { and } g \text { being associated to } S y s\end{array}$ & \\
and $\left.S y s_{g}\right)$. & \\
The system $S y s_{f}$ may have feedback information values out- & Req.4 \\
side $[m, M]$. & \\
\hline At any time, in the progress mode, when using $S y s_{f}$, if the & Req.5 \\
feedback information value of the controlled system equals & \\
to $m$ or to $M, S y s_{f}$ must is stopped. & \\
\hline
\end{tabular}

\subsection{Refinement Strategy}

The substitution process is defined for replacing $S y s_{f}$ by $S y s_{g}$, both systems being described by the state-transitions system of Fig. 2. Following the approach defined in [10], the adopted refinement strategy consists of an abstract specification and three refinements: Definition of a Mode controller (MO), Introduction of the safety envelope (M1), Handling continuous behavior and dense time (M2) and Discretization of the continuous behavior (M3). 


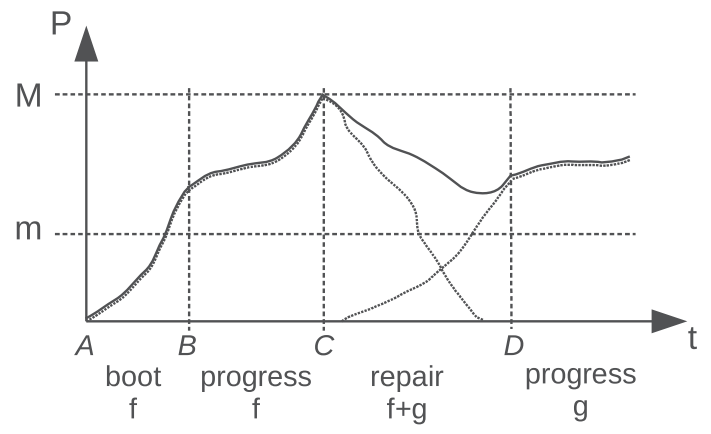

(a) Continuous system substitution

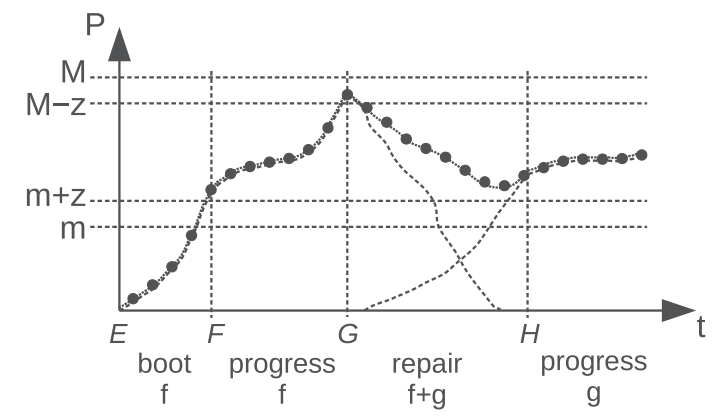

(b) Discrete system substitution

Fig. 3. Examples of the evolution of the function $f$

\subsection{The Event-B Models}

We describe the stepwise formal development of the models corresponding to the refinement strategy sketched above. All the Event-B models are available in [1].

- The required contexts. Contexts define the relevant concepts needed for our developments. The context $C_{-}$reals defines a set of theorems for positive reals, continuous and monotonic functions. This context uses the $R E A L$ type for real numbers defined in RealTheory (using the Theory plug-in for Rodin). The context $C_{-}$modes defines the constants $M O D E_{-} X$ representing the different system modes ( $F, G$ and $R$ for $S y s_{f}, S y s_{g}$ and Repair modes) belonging to the MODES set. The next two contexts ( $C_{-}$envelope and $C_{-}$margin) deal with the definition of the safety envelope. They define the intervals of safe values: $[m, M]$ in the continuous case, and $[m+z, M-z]$ with margin $z$ in the discrete case.

- The root machine: Definition of a Mode controller. Machine MO describes the reconfiguration state-transitions system depicted in Fig. 2. The modes are used in the events guards that allow the system to switch from one state to another. At initialization, $S y s_{f}$ is started $\left(M O D E_{-} F\right)$, it becomes active when the active variable is true (Sys $s_{f}$ ended the booting phase). When a failure occurs, progress of $S y s_{f}$ is stopped. The controller enters in the reparation mode $M O D E_{-} R$. Once the reparation is completed, the mode is switched to MODE_G and $\mathrm{Sys}_{g}$ enters into a progress state.

- First refinement: Introduction of the safety envelope. Machine M1 refines $M 0$. It preserves the behavior defined in $M 0$ and introduces two kinds of events: environment events (event name prefixed with $E N V$ ) and controller events (event name prefixed with $C T R L$ ) [10]. The ENV events produce the system feedback observed by the controller. Three continuous variables $f, g$ and $p$ are introduced. $f$ and $g$ record the feedback information values of $S y s_{f}$ and $S y s_{g}$ individually, while $p$ records the feedback value of both systems before, during and after substitution. $p$ corresponds to $f$ of $S y s_{f}$ in $M O D E_{-} F, g$ of $S y s_{g}$ in $M O D E_{-} G$ and $f+g$ of combined $S y s_{f}$ and $S y s_{g}$ in $M O D E_{-} R$ corresponding to the reparation after failure. Once the system has booted, $p$ must belong to the safety envelope in all the cases. The $C T R L$ events correspond to refinements 
of the abstract events of $M 0$. They modify the control variable active and $m d$ (the mode). The $E N V$ events observe real values corresponding to the different situations where $S y s_{f}$ and $S y s_{g}$ are running or when $S y s_{f}$ failed and $S y s_{g}$ is booting. This last situation corresponds to the reparation case.

- Second refinement: Continuous behavior and dense time. Machine M2 (refining M1) describes the continuous behavior (Fig. 3a). Once the modes and the observed values are correctly set, the next refining events are straightforward. They correspond to a direct reuse of the development of a correct discretization of a continuous function as done in [4]. Indeed, continuous variables $f_{c}, g_{c}, p_{c}$ and $m d_{c}$ corresponding to the functions $f, g, p$ and $m d$ to the modes in $M 1$ are introduced. A real positive variable now is defined to represent the current time. The gluing invariants (for example $p=p_{c}($ now )) connect the variables of M1 with the continuous variables at time now. In the same way, each event of $M 1$ is refined. A non-deterministic time step $d t$ is introduced and the continuous functions are updated by the $E N V$ events on the interval $[$ now, now $+d t]$ while now is updated to now $:=$ now $+d t$. The control CTRL events observe the value $p_{c}$ (now) to decide whether specific actions on mode $m d_{c}$ variable are performed or not. A detailed description of this refinement is given in [4].

- Third refinement: Discretization of the continuous behavior. Following [4], the discrete behavior is described in a Machine M3 (Fig. 3b). As mentioned in the context $C_{-}$margin, the margin $z$ is defined, such as $0<z \wedge m+z<$ $M-z$ and $M-m>2 \times z$. This margin defines, at the discrete level, the new safety envelope as $[m+z, M-z] \subset[m, M]$. The new discrete variables $f_{d}, g_{d}$, $p_{d}$ and $m d_{d}$ of $M 3$ are glued to $f_{c}, g_{c}, p_{c}$ and $m d_{c}$ of $M 2$. They correspond to discrete observations feedback of $f_{c}, g_{c}, p_{c}$ and $m d_{c}$. The discretization step is defined as $\delta t$. Each environment event corresponding to a continuous event is refined by three events. One discrete event starting a time interval $\delta t$, one for time intervals included in $\delta t$ and the next discrete event (third one) at end of $\delta t$. The second event may occur several times, a variant enforces it to be Zeno free. The discrete controller only observes the events at time $n \times \delta t$.

\section{Conclusion}

Modeling hybrid systems using Event-B was studied by several other authors $[7,8,10]$. In this paper, we have extended our work on system substitution to handle systems characterized by continuous models. First, we modeled system substitution at a continuous level, then we provided a discrete model for substitution which preserves the original continuous behavior. This work reused previous results we obtained on the correct system reconfiguration modeling and the correct discretization of continuous behaviors. By correctness, we mean the preservation of system information feedback in a safety envelope. The whole approach is supported by proof and refinement based on the Event-B method. Refinement proved useful to build a stepwise development which allowed us to gradually handle the requirements. Moreover, the availability of a theory of 
reals allowed us to introduce continuous behaviors which usually raise from the description of the physics of the controlled plants. All these models have been developed within the Rodin platform [3] and the developed formal models can be downloaded from [1].

\section{References}

1. Models. http://babin.perso.enseeiht.fr/r/ABZ_2016_Models/

2. Abrial, J.R.: Modeling in Event-B: System and Software Engineering, 1st edn. Cambridge University Press, New York (2010)

3. Abrial, J.R., Butler, M., Hallerstede, S., Hoang, T.S., Mehta, F., Voisin, L.: Rodin: an open toolset for modelling and reasoning in event-B. International Journal on Software Tools for Technology Transfer 12(6), 447-466 (2010). http://dx.doi.org/10.1007/s10009-010-0145-y

4. Babin, G., Aït-Ameur, Y., Nakajima, S., Pantel, M.: Refinement and proof based development of systems characterized by continuous functions. In: Li, X., et al. (eds.) SETTA 2015. LNCS, vol. 9409, pp. 55-70. Springer, Heidelberg (2015). doi:10.1007/978-3-319-25942-0_4

5. Babin, G., Ait-Ameur, Y., Pantel, M.: A generic model for system substitution. In: Romanovsky, A., Ishikawa, F. (eds.) Trustworthy Cyber Physical Systems Engineering. CRC Press Taylor \& Francis Group (2016)

6. Babin, G., Ait-Ameur, Y., Pantel, M.: Correct instantiation of a system reconfiguration pattern: a proof and refinement-based approach. In: 2016 IEEE High Assurance Systems Engineering Symposium, HASE 2016, Orlando, FL, USA, January 7-9, 2016. IEEE Computer Society Press (2016)

7. Banach, R.: Pliant modalities in hybrid Event-B. In: Liu, Z., Woodcock, J., Zhu, H. (eds.) Theories of Programming and Formal Methods. LNCS, vol. 8051, pp. 37-53. Springer, Heidelberg (2013)

8. Butler, M., Abrial, J.R., Banach, R.: From Action Systems to Distributed Systems: The Refinement Approach, chap. Modelling and Refining Hybrid Systems in Event-B and Rodin, p. 300. Taylor \& Francis, February 2016. http://www. taylorandfrancis.com/books/details/9781498701587/

9. Jastram, M.: Rodin User's Handbook (Oct 2013). http://handbook.event-b.org

10. Su, W., Abrial, J.R., Zhu, H.: Formalizing hybrid systems with Event-B and the Rodin platform. Sci. Comput. Program. 92(2), 164-202 (2014). http://www. sciencedirect.com/science/article/pii/S0167642314002482 